\title{
Epidemiologic Modeling of COVID-19 in Amhara Region, Ethiopia: Using The Best and Worst Scenarios Experienced in The World
}

Getachew Hailu ( $\sim$ getachewmph35@gmail.com )

Department of Epidemiology and Biostatistics, School of Public Health, College of Medicine and Health Sciences, Bahir Dar University

Tizta Tilahun

Department of Global Health and Population, Harvard T.H. Chan School of Public Health, Harvard University

\section{Gizachew Tadesse}

Department of Epidemiology and Biostatistics, School of Public Health, College of Medicine and Health Sciences, Bahir Dar University

\section{Denekew Bitew}

Department of Statistics, College of Sciences, Bahir Dar University

\section{Kassawmar Angaw}

Department of Epidemiology and Biostatistics, School of Public Health, College of Medicine and Health Sciences, Bahir Dar University

\section{Cherenet Mengistie}

Department of Epidemiology and Biostatistics, School of Public Health, College of Medicine and Health Sciences, Bahir Dar University

\section{Anemaw Asrat}

Department of Epidemiology and Biostatistics, School of Public Health, College of Medicine and Health

Sciences, Bahir Dar University

\section{Fentie Ambaw}

Department of Behavioral Science, School of Public Health, College of Medicine and Health Sciences, Bahir Dar University

\section{Yeshigeta Gelaw}

Department of ophthalmology, School of medicine, College of Medicine and Health Sciences, Bahir Dar University

\section{Getu Degu}

Department of Epidemiology and Biostatistics, School of Public Health, College of Medicine and Health Sciences, Bahir Dar University

\section{Essey Kebede}


Department of Epidemiology and Biostatistics, School of Public Health, College of Medicine and Health Sciences, Bahir Dar University

\section{Research Article}

Keywords: Morbidity, Mortality, Modeling, COVID-19

Posted Date: February 16th, 2021

DOl: https://doi.org/10.21203/rs.3.rs-190422/v1

License: (c) (i) This work is licensed under a Creative Commons Attribution 4.0 International License. Read Full License 


\section{Abstract}

Background: The ongoing pandemic of the novel coronavirus (SARS-CoV-2) is currently the first public health agenda. Citizens of resource-poor countries like Ethiopia could be most affected by the pandemic unless aggressive plans are implemented. Despite the prevention and control efforts being made at the national and regional levels, there is no evidence about the worst and best scenarios in the number of COVID-19 cases expected in the Amhara Regional. Besides, there were no efforts to develop predicting models of future values in COVID-19 anticipated cases expected based on the current global trend.

Objective: Forecast the latest epidemic situations by estimating and predicting cases and deaths of COVID-19 in the Amhara region.

Methods: This study employed models by using the expected number of COVID-19 cases and deaths in Amhara National Regional State using the worst scenarios faced in the world. Data were extracted using a checklist. Existing data on morbidity and mortality by age and sex were collected through a literature search strategy including PubMed/Medline and the Google scholar database. Besides, the Worldometer of the novel COVID-19 was used as a source of data. This study used MS-Excel spreadsheet program for data extraction. COVID-19modeling was made using Susceptible- Infected-Recovered (SIR), SusceptibleExposed Infected-Recovered (SEIR), and WHO DisMod II software.

Result: Without any pharmacological intervention, based on the SEIR model, the expected number of peak COVID-19 cases could reach about 3 million cases occurring on July $29^{\text {th }}, 2020$. However, with an intervention that could reduce the transmission by $30 \%$, would push the peak period to 22 days (20 ${ }^{\text {th }}$ August). Using the Susceptible-Infected-Recovered model without non-pharmacologic interventions, the expected number of peak COVID-19 cases is 5,947,685, occurring on July 2, 2020.The peak number of Hospitalization is 820,781 , in need of ICU 279,541and deaths 53,529. With the DisModll modeling, outputs showed that males are more affected than females. Accordingly, the total incident cases in the region were estimated to be 837,348 (474, 809 male and 362,540 female). The expected number of total deaths was also estimated at 44,247 (30, 176 male and 14, 071 female). Also, the middle age group is more infected but less at risk of death. The recovery rate decreases drastically as age increase.

Conclusion: The discrepancies in findings from the three models used in this study could be driven mainly by data limitation in the Ethiopia context. However, our results from the different models highlight the need for more intervention on non-pharmacological interventions. Besides, highly influential parameters urge further study for better effective evidence-based decision making.

\section{Background}

Coronavirus is one of the large families of RNA viruses. It causes illness in animals and humans. Originated in China, Wuhan in December 2019 rapidly spread to the globe. Subsequently, the World Health Organization (WHO) declared the outbreak as a public health emergency of 
international concern on January 30,2020, and on March 11, 2020, as a global pandemic(1).Compared with SARS and MERS, COVID-19 has spread more rapidly due increased globalization and the epidemic's focus(2).

Based on the initial report on the first 41 cases of the COVID-19 outbreak, most (73\%) of patients infected with COVID-19 were males, with less than half (32\%) possessing underlying comorbidities of diabetes, hypertension, and cardiovascular disease. It also reported that the median age of cases was 49 years. Of the initial 41 patients infected, $27(66 \%)$ had been directly exposed to the Huanan seafood market, and the Case Fatality Rate was nearly $2 \%(3)$.

A study on the COVID-19 outbreak on the Diamond Princess Cruise ship using the SEIR model with an assumption of relatively homogenous mixing of all people onboard, they estimated the basic reproduction rate (Ro)to 14.8 based on calibrating the predicted cumulative number of infections to the observed cumulative number of infections among all people onboard.

Accordingly, the daily reproduction rate $(\beta)$ was estimated to be 1.48 . For the model, considering the infectious period (i) of COVID-19 to be ten days, in the situation of no removal, the incubation period or the latent period $(\mathrm{L})$, was estimated to be approximately five days (ranging from 2 to 14 days) (4).

A study on the real-time forecasts of the COVID-19 epidemic in China from February $5^{\text {th }}$ toFebruary $24^{\text {th }}$, 2020, the latest 5-day forecasts, generated on February 9, estimate an average of 34,509 to 34,596 total cumulative cases in Hubei by February 14.Based on cumulative reported cases as of February $9^{\text {th }}$ these estimates correspond with an average of 7409to 7496 additional cases in Hubei within the next 5 days. Ten days forecasts generated on February 9, estimated 36,854 to 37,230 cumulative cases, on average, in Hubei by February 19. These estimates correspond with an additional 9754 to 10,130 cases in Hubei on average in the next 10 days. Finally, the 15-day forecasts predict a cumulative reported case count between 37,415 and38,028 cases, on average, in Hubei by February 24(5).

The very well-known model for infectious diseases is SEIR, which was used by the study on COVID-19 outbreak on the Diamond Princess Cruise ship $(4,6)$.

As it was justified in the study of modeling the epidemic dynamics in China, since COVID-19 outbreak and control characteristics are distinct from existing infectious diseases, the existing epidemic models such as SIR and SEIR cannot be applied to describe the observed data directly. A model called Susceptible, Unquarantined infected, quarantined infected, confirmed infected (SUQC) model to characterize the dynamics of COVID-19 and explicitly parameterize the intervention effects of control measures, which is more suitable for analysis than other existing epidemic models(7).

However, to use the SUQC model for the Amhara region's case, there is a need to know at least the initial values for the four variables of the model. Among these variables, the number of cumulative confirmed infections, $C(t)$, is usually the only variable with daily observed data for model fitting and parameter inference. Besides, the initial value of susceptible individuals $S(t)$ is approximately equal to the population size. The initially confirmed infections $C(t)$ is the number of infected obtained from the 
region's official report. Other parameters and initial values, including the quarantine rate $(\gamma 1)$, the confirmation rate ( $\beta$ ), the initial number of un-quarantine infected cases $(\mathrm{Uo})$, and the initial number of quarantine infected cases (Qo) to be estimated by fitting the daily time series of confirmed infections to the model defined as $\hat{C}=f(\gamma 1 . \beta$, Uo, Qo). However, it is too early to use this model since the data for the daily confirmed infections of the region are inadequate, being only six reported at the time of the study conduct.

Furthermore, none of the models had incorporated age and sex as factors for both to acquire and die of COVID-19. Moreover, while DisMod II was developed from the perspective of Global Burden of Disease studies, it may be of use for epidemiologists and public health researchers or anyone else trying to estimate COVID-19 epidemiology. Therefore, before prediction, estimation of some variables and parameters are essential through an epidemiological disease modeling approach using WHO DisMod-II software considering one extreme scenario faced in the world as worst. However, this modeling study tried to use different modeling approaches despite their limitations, including SIR and SEIR. This study aims to forecast the epidemic situation of COVID-19 and estimate and predict cases and deaths of the Amhara region using the world's best and worst scenarios using SEIR, SIR, and DisMod-II.

\section{Methods}

\section{Study area and period}

This study was conducted to model the expected number of COVID-19 cases in the Amhara region using the worst scenarios faced in the world. By the year 2020, the population is approximately $22,189,999$ $(11,068,826$ males and $11,121,173$ females), of which $80.58 \%$ ( $40.73 \%$ males and $39.85 \%$ females) live in rural areas. Amhara region is one of the nine National Regional states in Ethiopia. The region is further divided into11administrative Zones (North Gondar, South Gondar, North Wollo, South Wollo, North Shewa, East Gojjam, West Gojjam, Awi, Wag Himra, Oromiya and Bahir Dar special Zone) and 164 administrative Woredas. This study was conducted from April 2020 to May 2020.

\section{Study design and Levels of Analysis}

This study followed a time-series type of study design based on the review of the global and the United States reports, Epidemiological studies, the Worldometer website mortality and morbidity reportsofCOVID-19.Amhara region's population size and mortality pattern by age and sex structure also employed to estimate and predict the expected number of COVID-19 cases and deaths. The analysis was done at three stages: first at total regional population, second at regional population by sex separately (male and female), and third at regional population by five years age group category.

\section{Model Overview}




\section{SIR Model}

The SIR model is the flows of people between three states: susceptible (S), infected (I), and resistant or recovery (R). In Susceptible-infection-recovery (SIR) Model, we shall by $S(t), I(t)$, and R(t) denote the number of susceptible, infectious, and recovered, respectively, in the population at time $t$. Furthermore, it is assumed that the population consists of a constant number of $\mathrm{N}$ individuals and at all times $S(t)+l(t)+R(t)=N$. Dr. Alex Hoyt develops the model in an excel template.

\section{SEIR Model}

The susceptible-exposed-infected recovered (SEIR) models the flows of people between four states: susceptible (S), exposed (E), infected (I), and resistant (R). The SEIR model extends the SIR model to include an exposed but non-infectious class. Dr. Alison Hill develops the model. This Shiny app uses an epidemiological model based on the classic SEIR model to describe the spread and clinical progression of COVID-19.

\section{The DisMod II disease model}

It is a straightforward model with just one prevalent state. There are two causes of death: disease and 'all other' causes, which are assumed to be independent. There are 4 transition hazards: incidence, remission, case fatality, and the 'all other mortality' hazard. These transition hazards are age-specific, but DisMod assumes them to be constant within a one-year age interval. A basic assumption of the model is that the 'all other' causes mortality for healthy and diseased people. Hence, the disease model is completely determined by the three transition hazards, such as incidence, remission, and case fatality. Under this assumption, the following three differential equations describe the model:

$$
\frac{d S_{a}}{d a}=i S_{a}+r C_{a}, \frac{d S_{a}}{d a}=-(f+r) C_{a}+i S_{a}, \frac{d D_{a}}{d a}=f C_{a}
$$

Where the three model parameters, representing the transition hazards, are: i: incidence, $\mathrm{r}$ : remission, $\mathrm{f}$ : case fatality, and the three states are: Sa: Number of healthy people at age a, Ca: Number of diseased people at age a, Da: Number of dead people at age a.

\section{Data source and Data analysis}

This study used several data sources. Extracted data including the clinical and transmission parameters requires for the first Susceptible-Exposed-infected-Recovered (SEIR) were from the code for creating the $R$ shiny application website https://alhill.shinyapps.io/COVID19seir/. This code is a SEIR model for COVID19 infection, including different clinical trajectories of infection, interventions to reduce transmission, and 
comparisons to healthcare capacity https://github.com/alsnhll/SEIR_COVID19. For the SusceptibleInfected-Recovered (SIR) model, took the clinical and transmission parameters data from the COVID-19 SIR modeling excel template done by Dr. Alex Hoyt accessed from https://1drv.ms/x/s!Aihonr5JPgsehKYeJ... website on May 14, 2020. For the third DisMod II model, extracted data from an existing data source of mainly the Worldometer website designed for COVID-19 case monitoring https://www.worldometers.info/coronavirus/. Besides, information on variables such as the number of populations and mortality by age and sex of the Amhara region obtained from the Regional Health Bureau. Data extracted from the Worldometer website contains information on i: incidence (number of new COVID-19 cases), r: remission (number of recovered from COVID-19), f: case fatality (number of individuals who died of COVID-19) by age and sex

https://www.worldometers.info/coronavirus/coronavirus-age-sex-demographics/. The data was extracted through the data extraction checklist. The checklist was designed in an MS-excel worksheet. The extracted data entered DisMod-II. Once all available data on COVID-19 have been collated, first, whether the observations are all internally consistent was assessed. Analyzing whether the separate sources of information on remission, incidence, and mortality are consistent was checked using a computer. DisMod-II is a computer software program developed for the GBD that allows the user to check if a set of assumptions on incidence, prevalence, remission, case-fatality rates and observed mortality numbers are consistent with one another. DISMOD-II allowed us to change the age number and size for input and output variables as we require. It was advantageous when the epidemiological parameters or study indicators obtained or extracted from different sources in age groups differ from the age groupings in which we want to present the results for the Amhara region. The incidence rate, case fatality rate, and remission rate of COVID-19 for the Amhara region were estimated as an output using the region's population size by age and sex structure through the DISMOD software using an indirect standardization method. Subsequently, the expected cumulative incident cases and deaths of the region is calculated by multiplying the population size by the estimated incidence rate and case fatality rate as an output for the observed worst (highest) scenario. Two additional models, such as SEIR and SIR, were done for comparison purposes of their output.

\section{Variables and parameters}

For the DisMod-II modeling, the variables used as input were the world's highest incidence rate, case fatality rate, and remission rate by age and sex reported daily since it started in the USA for the last three months (from February 15 to May 13,2020). In addition, the Amhara region's population size by age and sex structure and mortality pattern were the variables used in this model. Moreover, clinical and transmission parameters for the SEIR (Table 1below) and SIR models were used (Table 2below).

Table 1 Parameters included in SEIR model, Amhara Region, Ethiopia, 2020 


\begin{tabular}{|c|c|c|c|}
\hline \multicolumn{4}{|c|}{ Clinical parameters and transmission rate parameters } \\
\hline Model parameters & Value & Model parameters & Value \\
\hline Duration incubation period & $\begin{array}{l}5 \\
\text { days }\end{array}$ & $\begin{array}{l}\text { Case fatality rate (auto } \\
\text { computed) }\end{array}$ & $2.50 \%$ \\
\hline Duration of mild infection & $\begin{array}{l}6 \\
\text { days }\end{array}$ & Population size & $22,189,999$ \\
\hline$\%$ infections that are severe & $15 \%$ & Initial number infected & 1 \\
\hline $\begin{array}{l}\text { Duration of severe infection (hospital } \\
\text { stay) }\end{array}$ & $\begin{array}{l}6 \\
\text { days }\end{array}$ & $\begin{array}{l}\text { Transmission rate - mild } \\
\text { infections }\end{array}$ & per day \\
\hline$\%$ of infections that are critical & $5 \%$ & Mild infection (Ro3*.15) & 0.45 \\
\hline Duration critical infection (ICU stay) & $\begin{array}{l}8 \\
\text { days }\end{array}$ & Sever infection (Ro3*0.05) & 0.15 \\
\hline Death rate for critical infections & $50 \%$ & Critical infection $($ Ro3*0.03) & 0.09 \\
\hline
\end{tabular}

Table 2 Parameters included in SIR model, Amhara Region, Ethiopia, 2020

\begin{tabular}{|c|c|c|}
\hline $\begin{array}{l}\text { Model } \\
\text { parameters }\end{array}$ & Value & Definitions \\
\hline Contact Rate & variable & The number of susceptible peoples an infectious person contact. \\
\hline Transmissibility & $2.0 \%$ & $\begin{array}{l}\text { The probability that a contact between a susceptible person and an } \\
\text { infected person results in infection. }\end{array}$ \\
\hline $\begin{array}{l}\text { Duration of } \\
\text { Infectiousness }\end{array}$ & 10 & $\begin{array}{l}\text { The period of time (in days) an infected person can pass the } \\
\text { infection }\end{array}$ \\
\hline $\begin{array}{l}\text { \% Needing } \\
\text { Hospitalization }\end{array}$ & $13.80 \%$ & The proportion of infected people who require hospital care. \\
\hline $\begin{array}{l}\% \text { Needing ICU } \\
\text { Care }\end{array}$ & $4.70 \%$ & The proportion of infected people who require ICU care. \\
\hline Mortality rate & $0.90 \%$ & The proportion of infected people who die. \\
\hline
\end{tabular}

\section{Adjusting the input data}

The extracted and entered data in DisMod-II were smoothed using two techniques. The incidence rate and case fatality rate were smoothed or adjusted with a cubic spline interpolation technique since it was observed best fit than other methods. In contrast, the remission rate smoothed with two periods moving average. Finally, the adjusted input data were used to estimate the incidence rate, case 
fatality rate, remission rate by age, and sex. The prevalence and mortality rate from all other causes by age and sex were also estimated.

\section{Describing the age-sex pattern and temporal trend of COVID-19}

The next step in the analysis was to look into trends by an age-sex pattern of the expected number of new cases and deaths of COVID-19 in the Region. The incidence rate patterns, case fatality rate, and remission rate were described numerically and graphically by age and sex. Furthermore, the temporal trend of COVID-19 is described using SEIR and SIR models.

\section{Results And Discussion}

Three models were used to estimate deaths and cases in the Amhara region. Each of them has their strength and limitations. The result obtained from each modeling technique was presented and discussed as follows:

\section{Susceptible-Exposed-Infected-Recovered (SEIR) Model}

For this model, parameters mentioned above: clinical parameters, the transmission rate parameters, and simulation values by considering the context of the Amhara region.

Our projection shows that with no intervention, the peak day will be at the beginning of August $\left(150^{\text {th }}\right.$ day after a case with coronavirus is confirmed); the region will experience about 4,222,172cases. On the 100th day, the day will be 21st June; the cases will reach 21, 917,130. In terms of the population exposed, the peak day will be on July $29^{\text {th }}$ with $3,013,605$ cases. After putting the different parameters in the model, findings indicate mild infection will be high in the region at 140 days, 31 st July with $3,167,679$ cases. It is estimated that people to be infected severely and critically in July will be the highest in experiencing $3,310,069$ cases and 142,548cases, respectively. After 125 days, the region will start having people recovered from the pandemic, on that day ( $16^{\text {th }}$ July) with $1,694,316$. The worst side of this pandemic is its fatality rate; the number of deaths that will be experienced high in the region estimated will be on the $160^{\text {th }}$ day on August $20^{\text {th }}$ with 273,458 deaths (Figure 1 ).

Even though the model predicts the peak number of severe, critical, and recovered cases could be occurred in July, what observed in the region in July was among 33, 166 individuals tested for Covid-19, about 642 and 478 were found test positive, and recovered from Covid-19 respectively. In addition, contrary to the predicted high number of deaths in August, the observed total death in August was 25 in the region. These contradicting findings might be due to mainly two possible reasons: first, the testing capacity of the region was so limited, particularly during the start of the pandemic, this, in turn, might miss infections and deaths from Covid-19; second, took the clinical and transmission parameters used in 
the model from other countries study estimates, which might not represent the developing countries' including Ethiopia's context.

To help the evaluation, we generated different scenarios. We tested the potential effects of intervention strategies to control or reduce social contacts. As literature shows, some public health interventions could curb the transmission of the pandemic in different degrees (8). For this study, we used an assumption of the region's implementing of intervention strategies, if able to reduce $30 \%$ of the virus's transmission; we estimated the following situations that the region might experience.

The results of the scenario with a $30 \%$ reduction in transmission describe the peak day will be at 222 days, on 21 st October with 2,407,042 cases (Figure 2). A drastic decrease in the number of cases compared to no intervention model if we increase the days of peak case burden by more than two months. A reduction in the reproduction rate will help in slowing the community transmission.

The result of the scenario with a $40 \%$ reduction in transmission was a decrease of about 2 million cases and able to increase the actual daily protection rate around 12 times. The peak day (December $15^{\text {th }}$ ) will be pushed by beyond four months, compared to the model with no intervention. It can be achieved by reducing both infection and death if strict public health intervention measures are implemented in the region (Figure 3).

\section{Susceptible-Infected-Recovered (SIR) Model}

\section{Number of Susceptible, Infected and Recovered Cases for COVID-19 if no Non-Pharmacologic intervention is implemented}

With and without non-pharmacologic intervention (NPI) the susceptible-Infected-Recovered (SIR) model was the most commonly used modeling technique for infectious diseases. Based on the SIR model the expected number of cases by date is shown in Figure 4 below. Without NPI that was designed in Excel as SIR modeling template and taking the parameters shown in Table 2 above as well as the total population of the Amhara region $(22,189,999)$ to be susceptible the predicted number of peak COVID-19 cases in the region was estimated to be $5,947,685$.

This finding emphasizes the peak date of occurrences of cumulative cases, need for hospitalization, and ICU care and death; accordingly, the above peak figures all were expected to be occurring on the same date on July 2, 2020. This prediction is without any non-pharmacologic interventions targeting in minimizing the daily contact rate such as school closure, physical distancing, self-isolation, quarantine, and lockdown.

\section{Number of cases in need of Hospitalization and ICU care and Death from COVID-19 if no Non- Pharmacologic Intervention is implemented}

Assuming that there are no interventions made in the region, the peak number of cases in need of hospitalization was expected to be 820,781 occurring on the date July 2, 2020. Regarding the maximum 
number of cases in need of ICU care, it was expected to be 279,541 the same date of peak hospitalization, July 2,2020 , and on the same date, the maximum number of death $(53,529)$ is expected (Figure 5).

\section{Number of Susceptible, Infected and Recovered Cases for COVID-19 when a Non-Pharmacologic intervention (NPI) is implemented}

If community interventions mainly focusing on the reduction of contact rate are applied, such as school closure and stay at home lockdown with an assumption of $80 \%$ reduction in contact rate (3.35) for at least six weeks from $4 / 15$ to $5 / 30$, the peak expected number of infected cases would be shifted from July 2, 2020, to August 27, 2020 (Figure 6).

\section{Number of cases in need of Hospitalization and ICU care and Death from COVID-19 with a Non- Pharmacologic Intervention (NIP)}

When a community intervention of school closure and lockdown are applied with an assumption of an $80 \%$ reduction in contact rate (3.35) from $4 / 15$ to $5 / 30$; the peak expected number of cases needing hospitalization and ICU care, and the peak number of deaths would be shifted from July 2, 2020, to August 27, 2020 (Figure 7).

\section{Number of Susceptible, Infected and Recovered Cases for COVID-19 with a Non-Pharmacologic intervention (NPI and reactive approach)}

If a non-pharmacologic intervention with a reactive approach is applied, targeting an $80 \%$ reduction of the contact rate during the first ten weeks (from April 15/2020 to June 30/2020) followed by a $20 \%$ reduction of the contact rate during the next 8 months (from July 1/2020 to March 12/2021) one can expect the following results. The peak number of infected cases would be $4,051,875$ on November 10/2020 (Figure 8).

With the same consideration of the NPI mentioned above, the peak number of cases needing hospitalization would be 559,159, while those needing ICU care are 190,438 . The estimated death is 36,467 on November10, 2020 (Figure 9).

If the NPIs are implemented successfully one can see how the date for the peak number of cases, needing hospitalization and ICU care as well as, death could be shifted from being early to late occurrences. This mechanism of delaying the outbreak in turn assists to develop the health institutions' capacity and to have preparedness for emergency response. However, such kinds of NPI couldn't be implemented sustainably due to their socio-economic impact hence releasing the restrictions and reopening schools is mandatory. The questions such as when and how the restrictions to be released depend on the levels of healthcare capacity and the preparedness for emergency response. Therefore, another alternative approach such as NPI of some duration taking the capacity developing time in to consideration with a reactive reduction is required. 
If NPIs and a reactive reduction in the contact rate is implemented, keeping all other parameters constant and assuming an $80 \%$ reduction in Contact Rate (3.35) for ten weeks (from April 15 to June 30,2020$)$ and then a reactive reduction of $20 \%$ (10.72) for the rest of six months, two important results were observed. The first was the epidemic curve could be flattened as a result the peak number of infected cases reduced from 5, 947, 685 cases to 4,051,875, those in need of Hospitalization decreased from 820,781 to 559,159 , needing ICU care from 279,541 to 190,438 and death from 53,529 to 36,467 . The second observation was a pronounced dalliance in the epidemic from July 2 to November 10,2020. These observations are essential findings that may inform us if this approach is used. It is possible to minimize the risk of acquiring the diseases and dying from it in one hand and get enough time to build capacity and prepare for the emergency response. Despite the important role of NPI in flattening the epidemic curve, low testing coverage and detection rate in the region could result in the hidden epidemic because onward transmission from asymptomatic and undetected infections accounts for $65 \%$ infectiousness (9).The peak day determines the duration of the epidemic, which is also dependent on our testing capacity. Testing campaigns can reduce the infection peak because the diagnosed population enters quarantine or treatment center and is less likely to affect the susceptible people(10). Moreover, on the month November, the observed data showed that among 165,498 individuals who tested for Covid-19, about 6,125 were found test positive, 3,226 recovered, and 89 died.

DisMod epidemiological diseases model

We used this model by considering the worst-case scenario to estimate the expected number ofCOVID-19 cases and deaths. Using the USA COVID-19 infected cases and deaths data by age and sex, it was tried to estimate the regional incidence, case fatality, and recovery rate in DisMod-II software with an indirect standardization method to the region's population size by age and sex. Accordingly, the expected number of infected cases and deaths were calculated by age and sex and presented as follows. Below is the male population graph on COVID-19 cumulative incidence rate, case fatality rate, and remission rate. In the graph, one can observe how the case fatality rate increases as age increases and the remission rate decreases as age increases, whereas the incidence rate remains more or less constant, except it is very low at an early age and low at an older age. Moreover, during an epidemic for an emerging disease not occurred previously, can observe that the cumulative incidence rate is more or less equal to the prevalence rate. This pattern is the same for both males and females though females have lower incidence and case-fatality rates compared to males at similar age while females have a higher remission rate compared to males (Figure 10 and Figure 11).

One can also estimate the expected number of COVID-19 cases, deaths, and recoveries overall or a specific age group by sex in the region using Table 3 and Table 4 . The total annual cumulative incident cases in the region were estimated to be $837,348(474,809$ male and 362,540 female cases). The expected number of total deaths in the region were also estimated at $44,247(30,176$ male and 14,071 female deaths). 
The Covid-19 data observed in the region so far showed that among 243, 638 total tested peoples, about 6760 were positive for Covid-19 and 3, 552 recovered while 125 deaths were registered. This observation is completely contradicting what was forecasted using the DisMod-II. It might be due to both the region's capacity for testing and the unrepresentative initial rates of the variables taken and used for the model.

The annual cumulative incidence rate and case fatality rate in Table below one can estimate the expected number of COVID-19 cases among the Amhara region's male population, and it is $4.2896 \%$ of $11,068,826$ male population that is estimated to be 474,809 .In addition, the expected number of COVID-19 deaths among the male population of the Amhara region is $6.3553 \%$ of the expected male COVID- 19 cases (474, 809 ) is estimated to be 30,176 cases.

Table 3 COVID-19 expected rates of new cases, deaths and remission among males by 5 years age group in Amhara Region, Ethiopia, 2020. 


\begin{tabular}{|c|c|c|c|c|c|}
\hline \multicolumn{6}{|c|}{ Males, Disease: COVID-19U (Rates * 100), sex: Males } \\
\hline \multicolumn{6}{|c|}{ Written 5/25/2020, 1:03:52 AM } \\
\hline Age & $\begin{array}{l}\text { Incidence } \\
\text { (rates * 100) }\end{array}$ & $\begin{array}{l}\text { Prevalence } \\
\text { (rates * 100) }\end{array}$ & $\begin{array}{l}\text { Remission } \\
\text { (rates * 100) }\end{array}$ & $\begin{array}{l}\text { Case fatality } \\
\text { (rates * 100) }\end{array}$ & $\begin{array}{l}\text { Mortality } \\
\text { (rates * 100) }\end{array}$ \\
\hline $0-4$ & 0.0044 & 0.0041 & 99.34 & 0.0303 & 0 \\
\hline $5-9$ & 0 & 0.0003 & 99.34 & 0.0389 & 0 \\
\hline $\begin{array}{l}10- \\
15\end{array}$ & 0.8692 & 0.5259 & 99.34 & 0 & 0 \\
\hline $\begin{array}{l}15- \\
19\end{array}$ & 3.9207 & 3.2261 & 99.34 & 0.0086 & 0.0003 \\
\hline $\begin{array}{l}20- \\
24\end{array}$ & 6.7598 & 6.2527 & 99.34 & 0.3143 & 0.0198 \\
\hline $\begin{array}{l}25- \\
29\end{array}$ & 7.8523 & 7.7006 & 99.34 & 1.2365 & 0.0953 \\
\hline $\begin{array}{l}30- \\
34\end{array}$ & 7.7227 & 7.6525 & 99.3365 & 3.0425 & 0.2326 \\
\hline $\begin{array}{l}35- \\
39\end{array}$ & 7.5197 & 7.2371 & 99.2863 & 5.8019 & 0.4196 \\
\hline $\begin{array}{l}40- \\
44\end{array}$ & 7.9559 & 7.3171 & 99.0899 & 8.9852 & 0.6582 \\
\hline $\begin{array}{l}45- \\
49\end{array}$ & 9.1516 & 8.1105 & 98.8643 & 11.8817 & 0.9663 \\
\hline $\begin{array}{l}50- \\
54\end{array}$ & 10.3517 & 9.1862 & 98.2221 & 13.7892 & 1.2689 \\
\hline $\begin{array}{l}55- \\
59\end{array}$ & 10.0143 & 9.2429 & 97.3381 & 14.1489 & 1.3056 \\
\hline $\begin{array}{l}60- \\
64\end{array}$ & 8.1336 & 7.9319 & 94.7431 & 13.5075 & 1.0671 \\
\hline $\begin{array}{l}65- \\
69\end{array}$ & 6.6564 & 6.5326 & 91.7705 & 13.8079 & 0.8999 \\
\hline $\begin{array}{l}70- \\
74\end{array}$ & 6.4137 & 6.3709 & 83.9362 & 17.1405 & 1.0926 \\
\hline $\begin{array}{l}75- \\
79\end{array}$ & 6.528 & 6.4765 & 78.8656 & 23.1684 & 1.5008 \\
\hline $80+$ & 6.498 & 6.7235 & 65.67 & 26.9479 & 1.8199 \\
\hline All & 4.2896 & 3.9548 & 97.5812 & 6.3553 & 0.2514 \\
\hline
\end{tabular}


Focusing on the annual cumulative incidence rate and case fatality rate, it is estimated that the expected number of COVID-19 cases among the Amhara region's female population is $3.2599 \%$ of $11,121,173$, which is 362, 540 (Table 4). Also, the expected number of COVID-19 deaths among the region's female population is $3.881 \%$ of expected female COVID-19 cases $(362,540)$, which are 14,071 .

Table 4 COVID-19 expected rates of new cases, deaths and remission among Females by 5 years age group in Amhara Region, Ethiopia,2020. 


\begin{tabular}{|c|c|c|c|c|c|}
\hline & \multicolumn{5}{|c|}{ DisMod II input and output, database COVID-19 } \\
\hline & \multicolumn{5}{|c|}{ Females, Disease: COVID-19U (Rates * 100), sex: Females } \\
\hline & \multicolumn{5}{|c|}{ Written 5/25/2020, 1:08:07 AM } \\
\hline Age & $\begin{array}{l}\text { Incidence } \\
\text { (rates * } \\
100)\end{array}$ & $\begin{array}{l}\text { Prevalence } \\
\text { (rates * } \\
100)\end{array}$ & $\begin{array}{l}\text { Remission (rates * } \\
100 \text { ) }\end{array}$ & $\begin{array}{l}\text { Case fatality (rates * } \\
\text { 100) }\end{array}$ & $\begin{array}{l}\text { Mortality (rates * } \\
\text { 100) }\end{array}$ \\
\hline $0-4$ & 0.003 & 0.0028 & 99.76 & 0.0188 & 0 \\
\hline $5-9$ & 0 & 0.0002 & 99.76 & 0.0241 & 0 \\
\hline $10-14$ & 0.6309 & 0.3806 & 99.76 & 0 & 0 \\
\hline $15-19$ & 2.8667 & 2.348 & 99.76 & 0.0054 & 0.0001 \\
\hline $20-24$ & 4.9826 & 4.5889 & 99.76 & 0.1945 & 0.009 \\
\hline $25-29$ & 5.8098 & 5.691 & 99.7551 & 0.7647 & 0.0436 \\
\hline $30-34$ & 5.7111 & 5.688 & 99.7225 & 1.8813 & 0.1069 \\
\hline $35-39$ & 5.552 & 5.4168 & 99.6905 & 3.5875 & 0.1942 \\
\hline $40-44$ & 5.8725 & 5.5221 & 99.6056 & 5.5553 & 0.3072 \\
\hline $45-49$ & 6.7667 & 6.1735 & 99.533 & 7.3453 & 0.4547 \\
\hline $50-54$ & 7.6743 & 7.0244 & 99.3477 & 8.5236 & 0.5997 \\
\hline $55-59$ & 7.4254 & 7.0686 & 98.8287 & 8.7458 & 0.6172 \\
\hline $60-64$ & 6.0132 & 6.0072 & 97.1273 & 8.3496 & 0.4995 \\
\hline $65-69$ & 4.9049 & 4.8886 & 95.3137 & 8.5339 & 0.4161 \\
\hline 70-74 & 4.7284 & 4.6734 & 90.7169 & 10.591 & 0.4951 \\
\hline $75-79$ & 4.8154 & 4.7272 & 88.1846 & 14.3183 & 0.6769 \\
\hline $80+$ & 4.8035 & 4.7768 & 82.56 & 16.6571 & 0.797 \\
\hline $\begin{array}{l}\text { All } \\
\text { ages }\end{array}$ & 3.2599 & 3.0378 & 98.9243 & 3.881 & 0.1179 \\
\hline
\end{tabular}

With the application of DisMod-II considering the United States of American's (USA) data by age and sex, the annual cumulative incidence rate of COVID-19 was estimated at 837,348 . This result is based on the age-sex specific rates observed in the USA after a community transmission started on May 13,2020 Worldometer's report. As clearly described it in the result part, one can look at the age-sex pattern of COVID-19 cumulative incidence, Case fatality and recovery rates. 
Unlike the above two models (SIR and SEIR), this model result emphasizes indicating the most affected group of peoples than the peak of several expected cases by date. Moreover, it could not detect the impact of NPI on the magnitude and in delaying peak date of occurrences of COVID-19 with this model. However, contrary to the other models, it indicates the high-risk groups (at least by age and sex) with their number expected to be infected, recovered, and dead; this, in turn, aid deciding about preventing and controlling the pandemic in the region. Accordingly, it showed that cumulative incidence is very low at lower age groups (less than 15 years old) and low at higher age group (greater than 65 years old); however, it is highest among the working-age groups (from 15 to 65 years old). It might be due to their contact rate per day. Since they are mostly unemployed young and old dependents having limited movements, peoples at the lowest and highest age groups have lower average contact rates per day compared to the middle working age groups. It might pronounce if measures like school closure were made. Even though the acquiring probability is higher in the middle age group, the fatality rate is very high among those sixty and above age group people with the lowest recovery rates. This idea is supported by the CDC report that most USA deaths are above $75 \mathrm{~s}$ (11). Similarly, the WHO's $11^{\text {th }}$ $14^{\text {th }}$ weekly reports indicated that 60 and above age group peoples are at high risk of death from COVID19 than the younger age groups(12).In this modeling, it was tried to explore the age-adjusted gender variation through an indirect method of adjustment. As a result, males' cumulative incidence rate is 1.32 times higher than females at 4,290 per 100, 000 and 3,260 per 100, 000 respectively. Similarly, the overall male death rate is 1.6 times higher than females at 6,355 per 100,000 and 3,881 per 100,000 respectively. This gender variation in the death rate from COVID-19 is in line with the CDC's report of COVID-19 much more fatal for men, especially taking age into account, it indicated that in New York, the overall male death rate is 1.7 times higher than the female death rate at 228 per 100,000 and 134 per 100,000 , respectively.

\section{Conclusion And Recommendation}

The SEIR and SIR models inform us that if non-pharmacologic interventions are applied, such as physical distancing, school closure, and others aggressively, we can delay the epidemic peak day and flatten the epidemic curve, better preparedness for the emergency response and developing health care capacity. The DisMod-II modeling informed us about the maximum annual cumulative number of COVID-19 cases that can be occurred by age and sex. Moreover, it clearly showed us the most affected groups of people; thus, though the middle age groups were more at risk for acquiring the infection, they are less at risk of death than the older ones. Similarly, males were more at risk of death than females. This information could help officials for decision making.

The study can guide decision-makers in deciding on the optimal scheduling of intervention possible to delay the peak of infection. The delay in the time of peak infection gives room for better preparedness. The best combination of interventions could less burden the health facilities with the implementation of NPIs. Adequate implementation of NPIs helps in reducing death and flattening the curve. The model predictions could help in applying effective mitigation and suppression strategies. More effective use of 
strategies based on the caseload and other factors besides, the risk of specific contexts fastens in combating the pandemic is crucial in controlling the pandemic.

\section{Declarations}

\section{Ethics approval and consent to participate}

Ethical clearance was obtained from ethical review committee of Bahir Dar University, College of Medicine and Health Sciences, School of Public Health and Department of Biostatistics and Epidemiology. Consent to participate is not applicable since this study did not take data from individual person.

\section{Consent for publication}

Consent for publication is not applicable. This study did not take individual person's detail such as name, images, or videos.

\section{Availability of data and material}

The datasets generated and/or analyzed during the current study are available in the [ACCESSIBLE WEB LINKS TO DATASETS]

https://alhill.shinyapps.io/COVID19seir/ for SEIR model

https://github.com/alsnhll/SEIR_COVID19 for SEIR model

https://onedrive.live.com/view.aspx?

resid=1E0B3E49BE9E6828!70430\&ithint=file\%2cxIsx\&authkey=!ACcYayKvUyplrds for SIR model

https://www.worldometers.info/coronavirus/ for DisMod-II model

https://www.worldometers.info/coronavirus/coronavirus-age-sex-demographics/ for DisMod-II

\section{Competing interests}

No, I declare that the authors have no competing interests as defined by BMC, or other interests that might be perceived to influence the results and/or discussion reported in this paper.

\section{Funding}

No funding was received

\section{Authors' contributions}

G.H, T.T, G.T, D.B and C.M wrote the proposal, extract data, wrote the result and the manuscript. K.A, A.A, F.A, Y.G, G.D and E.K reviewed the proposal and the result. All authors reviewed the manuscript. 
Acknowledgments

The authors would like to thank, Bahir Dar University and Amhara regional health bureau for giving access of regional population data for analysis.

\section{References}

1. Burhan E, Prasenohadi P, Rogayah R, Isbaniyah F, Reisa T, Dharmawan I. Clinical Progression of COVID-19 Patient with Extended Incubation Period, Delayed RT-PCR Time-to-positivity, and Potential Role of Chest CT-scan. Acta Medica Indonesiana. 2020;52(1):80 \%@ 2338-732.

2. Yang S, Cao P, Du P, Wu Z, Zhuang Z, Yang L, et al. Early estimation of the case fatality rate of COVID19 in mainland China: a data-driven analysis. Ann Transl Med. 2020;8(4):128.

3. Noah C Peeri NS, Md Siddikur Rahman, Rafdzah Zaki, Zhengqi Tan, Saana Bibi, Mahdi Baghbanzadeh, Nasrin Aghamohammadi, Wenyi Zhang and Ubydul Haque. The SARS, MERS and novel coronavirus(COVID-19) epidemics, the newest and biggest global health threats: what lessons have we learned? International Journal ofEpidemiology, . 2020;0(0):1-10.

4. Rocklöv J SH, Wilder-Smith A COVID-19 outbreak on the Diamond Princess cruise ship: estimating the epidemic potential and effectiveness of public health countermeasures. Journal of Travel Medicine. 2020.

5. Roosa K, Lee Y, Luo R, Kirpich A, Rothenberg R, Hyman JM, et al. Real-time forecasts of the COVID-19 epidemic in China from February 5th to February 24th, 2020. Infect Dis Model. 2020;5:256-63.

6. Stehlé J, Voirin N, Barrat A, Cattuto C, Colizza V, Isella L, et al. Simulation of an SEIR infectious disease model on the dynamic contact network of conference attendees. BMC Med. 2011;9.

7. Zhao S, Chen H. Modeling the epidemic dynamics and control of COVID-19 outbreak in China. Quant Biol. 2020:1-9.

8. Regmi K, Lwin CM. Impact of non-pharmaceutical interventions for reducing transmission of COVID19: a systematic review and meta-analysis protocol. BMJ open. 2020;10(10):e041383 \%@ 20446055.

9. Chen Y, Wang A, Yi B, Ding K, Wang H, Wang J, et al. The epidemiological characteristics of infection in close contacts of COVID-19 in Ningbo city. Chin J Epidemiol. 2020;41(5):668-72.

10. Giordano G, Blanchini F, Bruno R, Colaneri P, Di Filippo A, Di Matteo A, et al. Modelling the COVID-19 epidemic and implementation of population-wide interventions in Italy. Nature Medicine. 2020:1-6 \%@1546-170X.

11. Reeves RV, Ford T. COVID-19 much more fatal for men, especially taking age into account. Brooking Series: Middle Class Memos. Published May 15, 2020. 2020.

12. Organization WH. COVID-19 weekly epidemiological update. 2020.

\section{Figures}




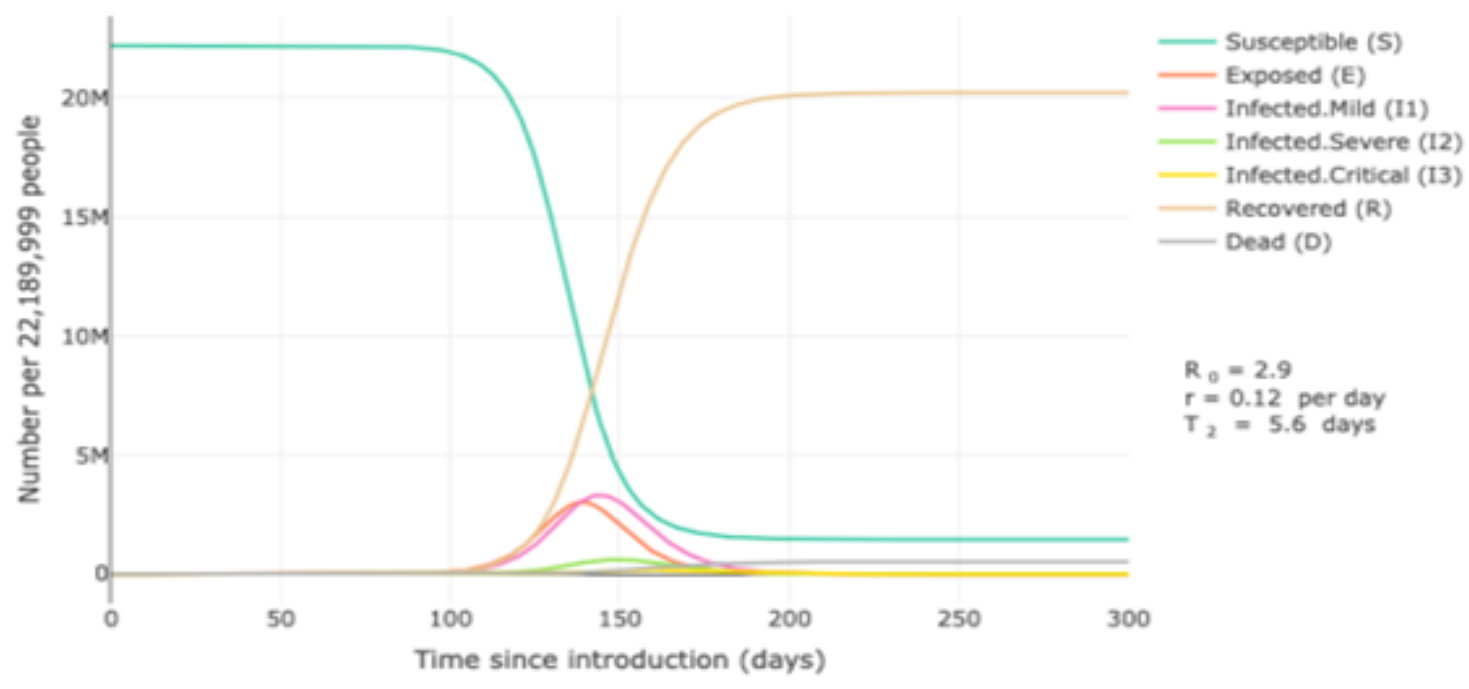

Figure 1

The SEIR model for Covid-19 with no intervention, Amhara Region, Ethiopia, 2020.

\section{Reduction in predicted COVID-19 after intervention}

Simulate the change in the time course of COVID-10 cases after applying an intervention

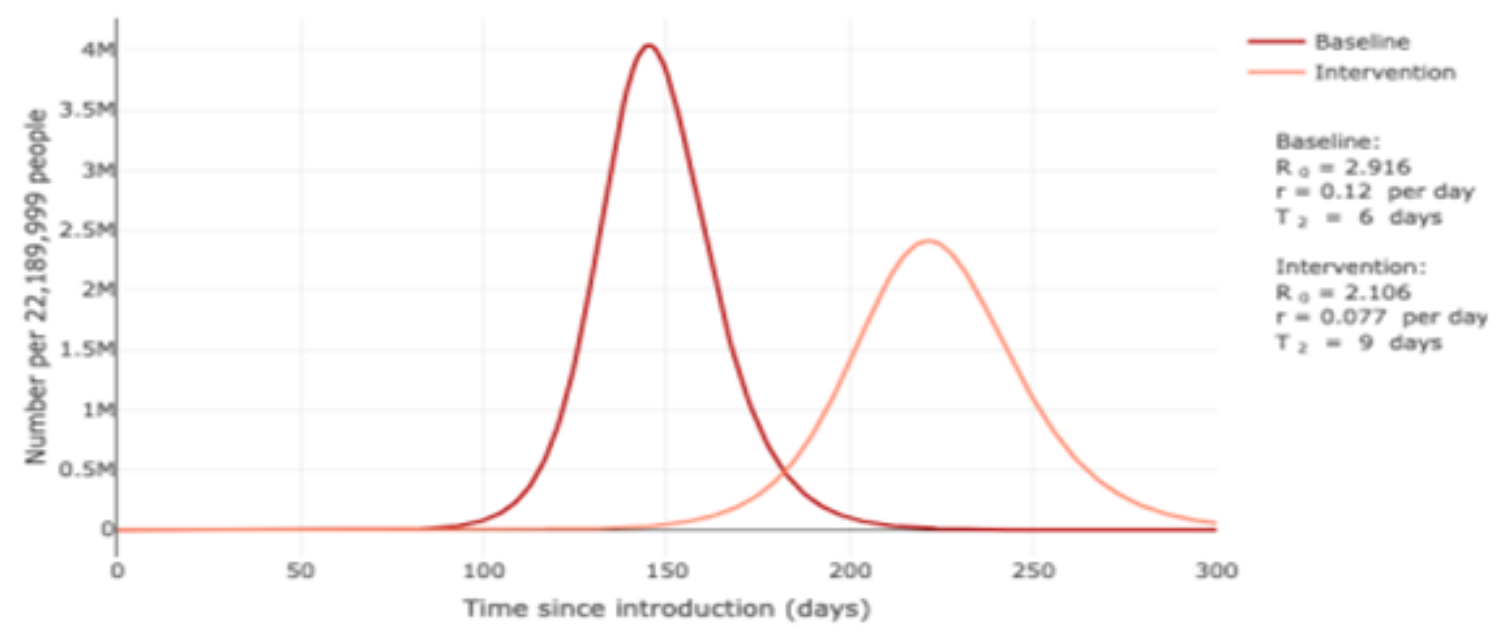

Figure 2

The model with 30\% reduction in transmission of the pandemic, Amhara Region, Ethiopia, 2020. 


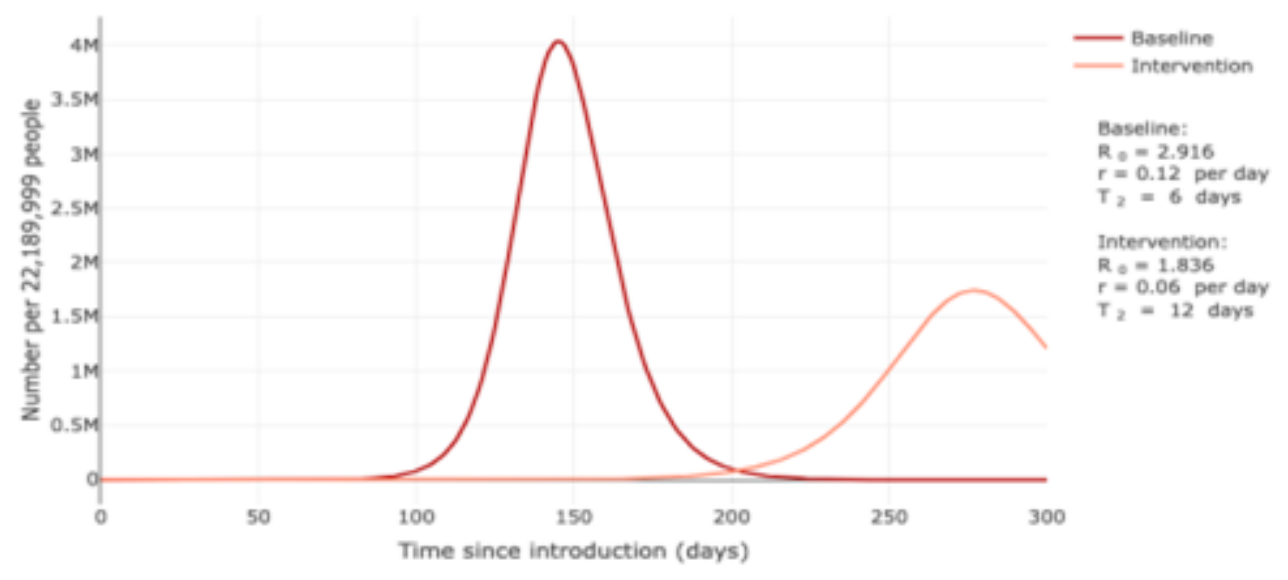

\section{Figure 3}

The model with 40\% reduction in transmission of the pandemic, Amhara Region, Ethiopia, 2020.

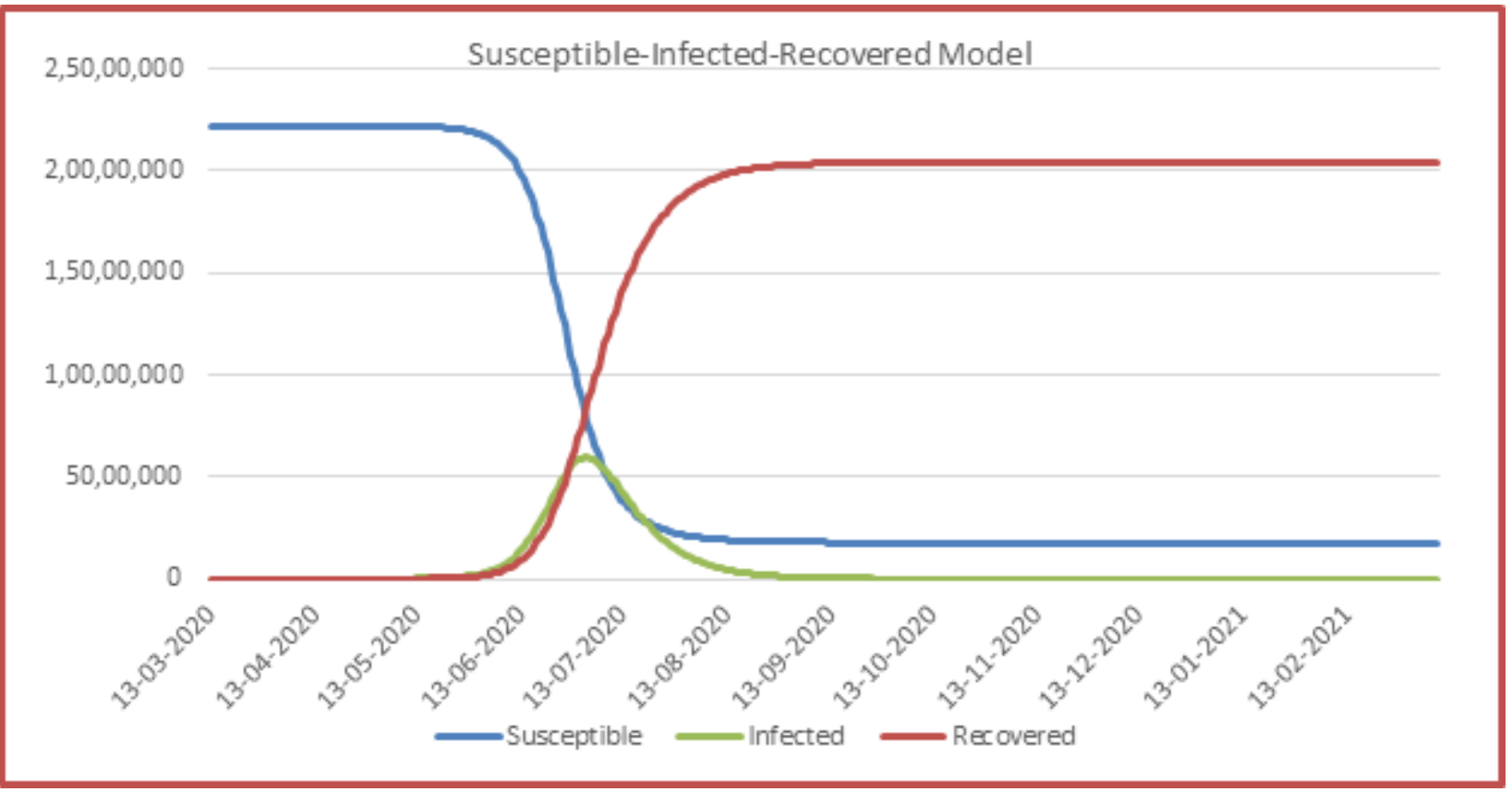

\section{Figure 4}

Expected Number of Susceptible-Infected-Recovered (SIR) cases for COVID-19 by date if no nonepharmacologic intervention model in Amhara Region, Ethiopia, 2020. 


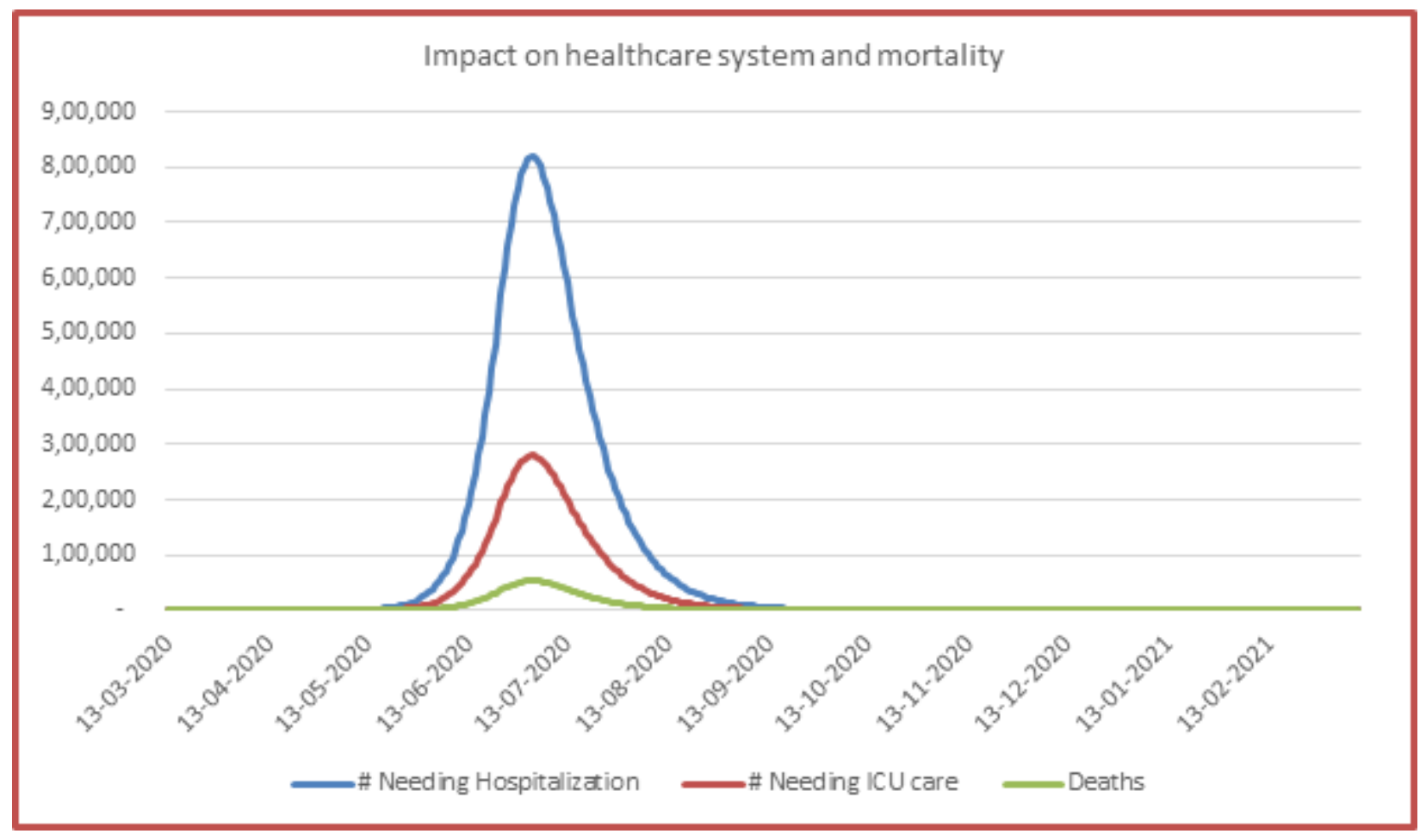

Figure 5

Expected Number of cases needing hospitalization and ICU care and death fromCOVID-19 by date in Amhara Region, Ethiopia, 2020.

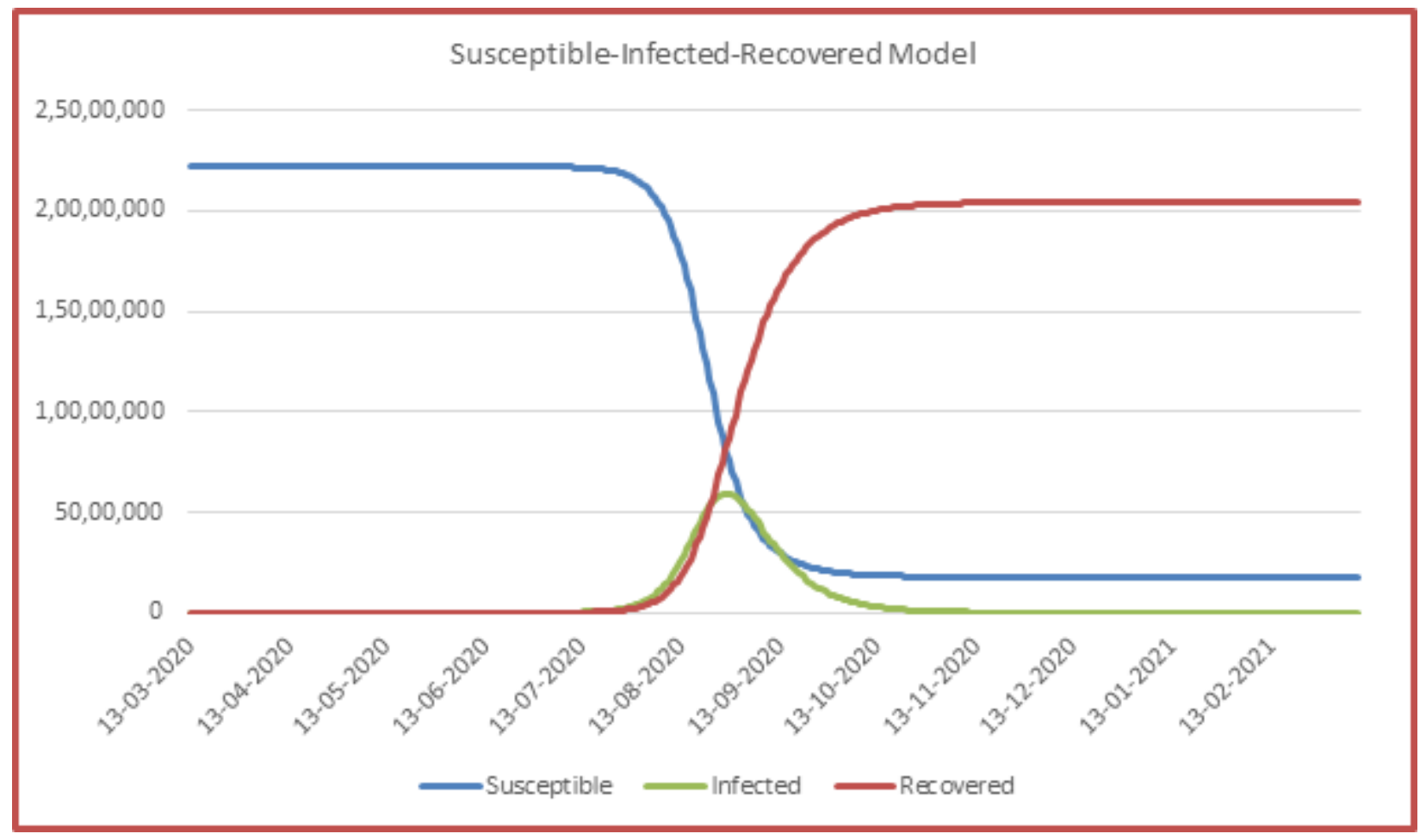

\section{Figure 6}

Expected Number of Susceptible-Infected-Recovered (SIR) cases for COVID-19 by date with a community intervention aimed at 80\% reduction of Contact rate in Amhara Region, Ethiopia, 2020. 


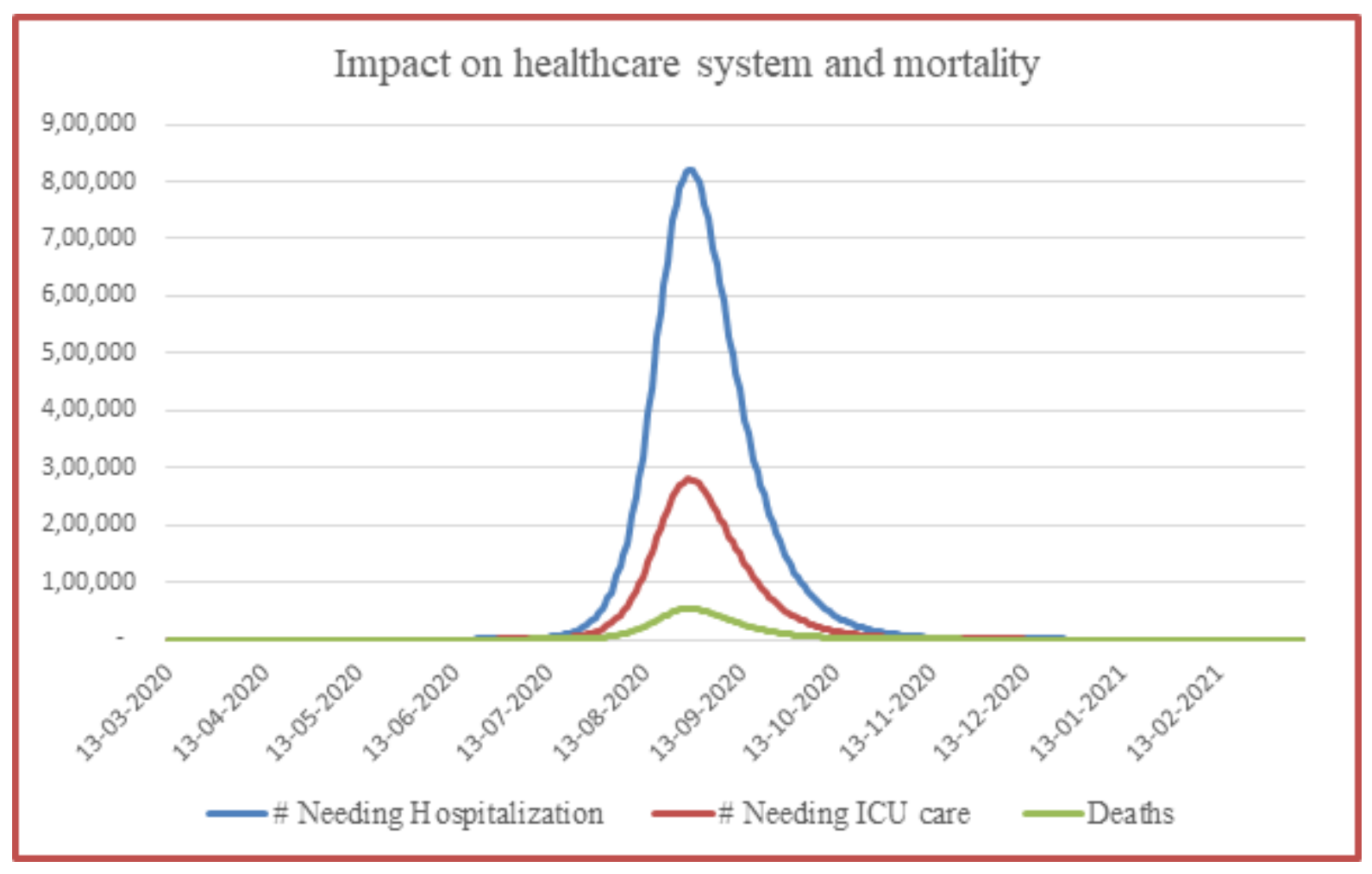

\section{Figure 7}

Expected Number of cases needing hospitalization and ICU care and death from COVID-19 by date with a community intervention aimed at 80\% reduction of Contact rate in Amhara Region, Ethiopia, 2020.

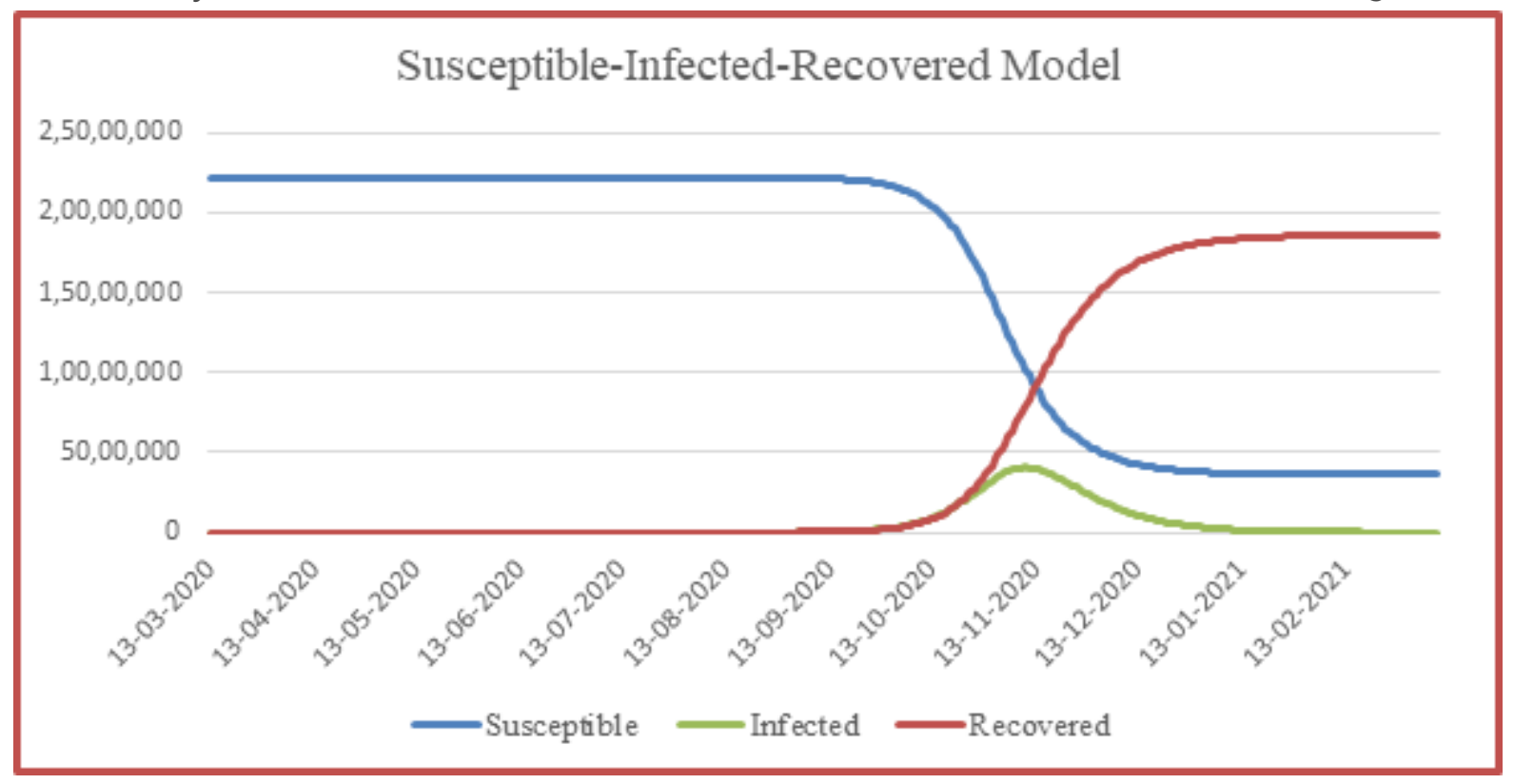

\section{Figure 8}

Expected Number of Susceptible-Infected-Recovered (SIR) cases for COVID-19 by date with an intervention aimed at $80 \%$ reduction of Contact rate in Amhara Region, Ethiopia, 2020. 


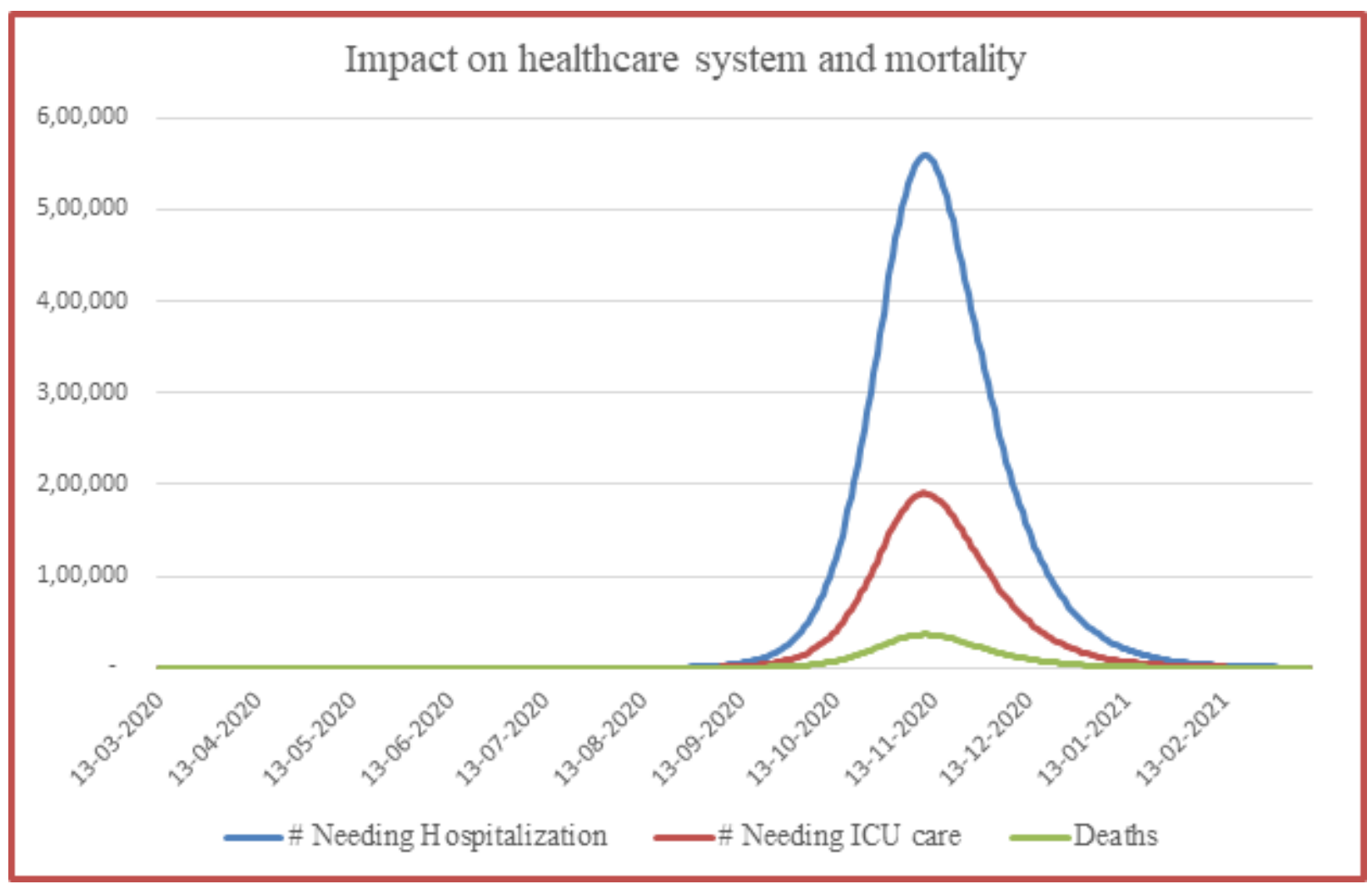

\section{Figure 9}

Expected Number of cases needing hospitalization and ICU care and death from COVID-19 by date with a community intervention aimed at $80 \%$ followed by $20 \%$ reduction of Contact rate in Amhara Region, Ethiopia, 2020. 


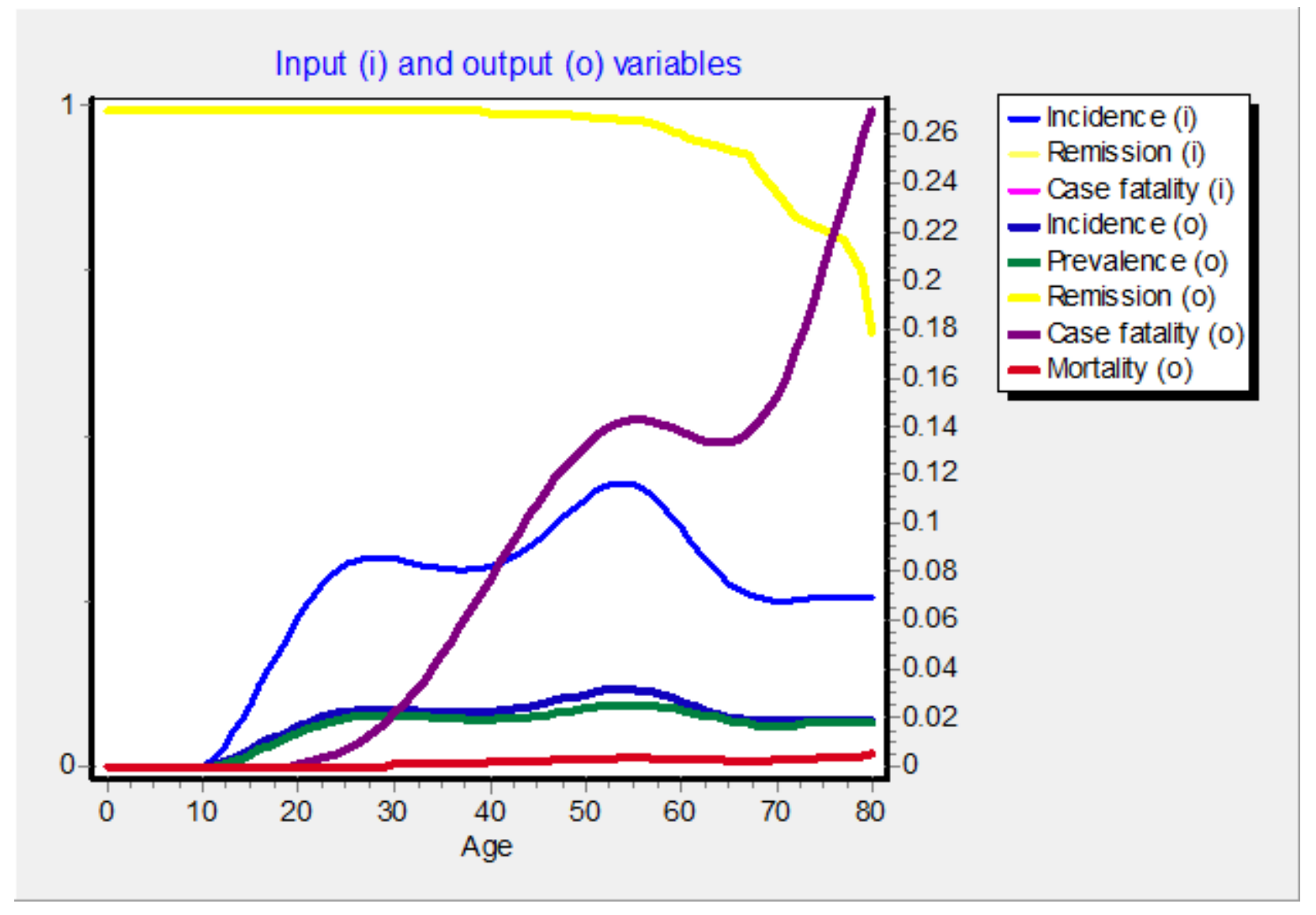

Figure 10

Expected incidence, recovery and case fatality rates from COVID-19 of males by Age in Amhara Region, Ethiopia, 2020. 


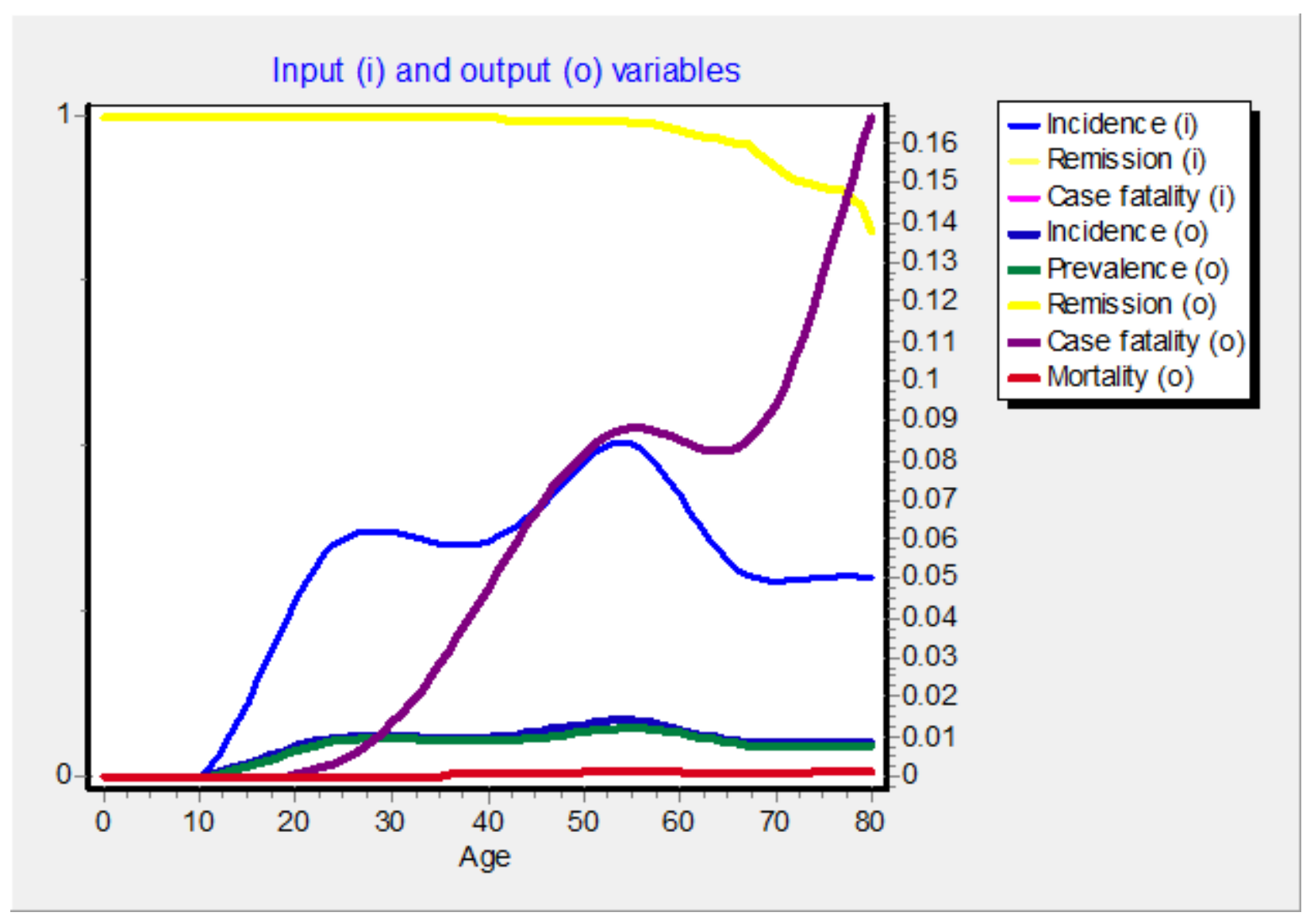

\section{Figure 11}

Expected incidence, recovery and case fatality rates from COVID-19 of Females by Age in Amhara Region, Ethiopia, 2020. 